\title{
Canine Nephrotoxicosis Induced by Melamine-Contaminated Pet Food in Italy
}

\author{
Monia COCCHI ${ }^{1}$, Marta VASCELLARI ${ }^{1)}$, Albino GALLINA ${ }^{1)}$, Fabrizio AGNOLETTI ${ }^{1}$, Roberto ANGELETTI ${ }^{1)}$ and \\ Franco MUTINELLI ${ }^{1) *}$
}

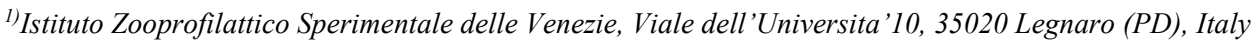

(Received 26 June 2009/Accepted 15 September 2009/Published online in J-STAGE 13 November 2009)

\begin{abstract}
Two young dogs belonging to the same kennel placed nearby Treviso (north-eastern Italy) died at the end of 2008 with clinical signs of renal failure. They were subjected to necropsy and were evaluated for histopathological and toxicological changes. Both the animals had same clinical signs and laboratory evidence of uremia. Post mortem investigations revealed severe nephrotoxicosis, associated with uroliths deposition within renal tubules and pelvis. The predominant crystal type was identical to those observed in the kidneys of animals involved in the 2004 and 2007 melamine-associated renal failure epidemic in Asia and US, providing evidence that they share the same causative agent. High doses of melamine were detected in the pet food administered to the dogs, likewise melamine was identified in renal tissue from one dead dog and in urine samples from both the animals. Therefore, a diagnosis of melamine-related nephrotoxicosis was made. To the author's knowledge this is the first report about melamine contamination of pet food from EU. KEY WORDS: canine, melamine, nephrotoxicosis, pet food.
\end{abstract}

Melamine is an organic base containing a relatively large quantity of nonprotein nitrogen. Melamine-related compounds include cyanuric acid, ammeline, and ammelide. Because of its high nitrogen content, melamine is a common additive in animal feeds in China [17]. Melamine is of no known nutrient value to dogs or cats. Addition of melamine and related compounds as ingredients in animal or human food is illegal in the United States and EU [17]. Melamine seems to have a wide margin of safety. If consumed, it is relatively nontoxic to man and animals. However, in rodents chronic exposure to melamine causes urolithiasis, and chronic irritation of the urothelium by uroliths induces urothelial hyperplasia and subsequent urinary bladder neoplasia. Melamine has also been reported to cause diuresis in rats and dogs, crystalluria in mice, rats, and dogs, and fatal uremia characterized primarily by crystalluria in sheep [17]. Apparently melamine and related compounds do not accumulate in the body. Instead, they are rapidly eliminated by the kidneys [20]. Recently it has been determined that a combination of melamine and cyanuric acid are lethal nephrotoxins in cats and dogs $[2,7,9,20,23]$. A combination of these two compounds is lithogenic in dogs. On March 16, 2007, Menu Foods Inc., a Canadian manufacturer of wet pet food, issued a recall of more than 60 million containers of cuts and gravy style pet food because of concerns about the effects of the product on the renal health of pets [3-5]. Food related acute renal failure was diagnosed in dogs and cats in veterinary clinics across the country. It was reported that at least 471 cases of renal failure had been reported in a 10-day period, and 104 dogs and cats had died [1]. Melamine in wheat gluten originating from China was later found in the recalled products [5].

\footnotetext{
* Correspondence to: Mutinelli, F., Istituto Zooprofilattico Sperimentale delle Venezie, Viale dell'Universita'10, 35020 Legnaro (PD), Italy.

e-mail: fmutinelli@izsvenezie.it
}

Aim of this study is to describe an episode of renal failure associated with consumption of melamine-contaminated pet food in dogs in Italy.

On November 2008, a 3-year-old, male, Maltese (Case No. 1) was submitted to the Treviso diagnostic service of the Istituto Zooprofilattico Sperimentale delle Venezie, for post-mortem examination. The dog belonged to the same kennel with other 15 dogs. Symptoms referred by dog's owner were abdominal pain, weakness, anorexia with feed refusal, vomiting, and depression. Serum biochemistry and urine analysis denoted renal failure (Table 1). In the urine sediment numerous, unstained round shaped crystals, with many lines radiating from their centres, were present. Scant calcium oxalate monohydrate, uric acid and ammonium urate crystals were also evident. No casts were observed. At necropsy bilateral yellowish discolouration of the inner part of the medulla with deposit of green yellowish uroliths $(0.1-0.3 \mathrm{~cm})$ in the medulla and renal pelvis was detected (Fig. 1). No gross lesions were observed in the ureters, while diffuse thickening of the urinary bladder wall was evident. Ulceration of the oral mucosa, haemorrhagic gastritis and enteritis, and diffuse peritoneal petechiae were also present. On opening the thoracic cavity, the lungs appeared collapsed with widespread miliary areas of paleness $(0.1 \mathrm{~cm}$ of diameter) with central emphysematous bullae, surrounded by atelectasic areas.

On December 2008, a 1-year-old, male, Lhasa Apso (Case No.2) belonging to the same breeder, died with symptoms of renal failure. Significant serum biochemical findings and urinalysis are reported in Table 1. Scant round shaped crystals and calcium oxalate monohydrate crystals were observed in the urine sediment, while no casts were observed. At necropsy nephrosis of the right kidney with uroliths morphologically similar to those described herein, and polycystic left kidney were recorded.

The 2 dogs were fed the same brand of dry pet-food man- 
Table 1. Laboratory findings

\begin{tabular}{lccc}
\hline \multicolumn{4}{c}{ Serum biochemical analysis } \\
Test & Case no. 1 & Case no. 2 & \\
\hline & \multicolumn{2}{c}{ Obtained Value } & Reference range \\
Blood urea nitrogen & $21.6 \mathrm{mmol} / \mathrm{L}$ & $70.9 \mathrm{mmol} / \mathrm{L}$ & $1.67-6.7$ \\
Creatinine & $380 \mu \mathrm{mol} / \mathrm{L}$ & $684 \mu \mathrm{mol} / \mathrm{L}$ & $44-133$ \\
Serum phosphorus & $4.02 \mathrm{mmol} / \mathrm{L}$ & $4.29 \mathrm{mmol} / \mathrm{L}$ & $0.84-2$ \\
Serum calcium & $1.65 \mathrm{mmol} / \mathrm{L}$ & $3.04 \mathrm{mmlo} / \mathrm{L}$ & $2.25-2.83$ \\
Albumin & $32 \mathrm{~g} / \mathrm{L}$ & $41 \mathrm{~g} / \mathrm{L}$ & $26-33$ \\
Globulin & $41 \mathrm{~g} / \mathrm{L}$ & $22 \mathrm{~g} / \mathrm{L}$ & $26-44$ \\
ALT (a) & $146 \mathrm{U} / \mathrm{L}$ & $75 \mathrm{U} / \mathrm{L}$ & $21-102$ \\
TBIL (b) & $0.2 \mathrm{mg} / \mathrm{dL}$ & $0.3 \mathrm{mg} / \mathrm{dL}$ & $0.0-0.9 \mathrm{mg} / \mathrm{dL}$ \\
Glucose & $9.8 \mathrm{mmol} / \mathrm{L}$ & $4.5 \mathrm{mmol} / \mathrm{L}$ & $3.61-6.55$ \\
\hline \multicolumn{4}{c}{ Urine analysis } \\
Test & Case no. 1 & Case no. 2 & \\
Specific gravity & 1.018 & 1.020 & Reference range \\
pH & 6 & 6 & $1.001-1.065(\mathrm{c})$ \\
Glucose & $150 \mathrm{mg} / \mathrm{dL}$ & $130 \mathrm{mg} / \mathrm{dL}$ & $5.5-7$ \\
Ketones & $0.0 \mathrm{mmol} / \mathrm{L}$ & $0.0 \mathrm{mmol} / \mathrm{L}$ & - \\
Bilirubin & Absent & Absent & - \\
Hematuria & ++++ & +++ & - \\
Protein & $100 \mathrm{mg} / \mathrm{dL}$ & $18 \mathrm{mg} / \mathrm{dL}$ & - \\
Leukocytes & Present & Present & - \\
Red blood cells & Present & Present & - \\
(a). ALT, alanine aminotransferase. (b). TBIL, total bilirubin. (c). & - \\
\hline
\end{tabular}

ufactured in Italy. The veterinarian reported that other dogs of the same kennel were showing clinical signs of renal failure.

Bacteriological and virological investigations were performed on intestine, liver and urine by routine laboratory tests. Samples of kidney, urinary bladder, lung, stomach, gut, and liver were fixed in $10 \%$ neutral-buffered formalin, routinely processed, embedded in paraffin, sectioned at approximately $5 \mu \mathrm{m}$, and stained with haematoxylin and eosin (H\&E); further sections of the kidney were stained by Oil Red O (ORO), a stain for lipids and plastics [12], and Von Kossa, a stain for phosphates, carbonates and oxalates of calcium, iron, and other ions [6].

Samples of two kinds of dry pet food, urine samples from dog Nos. 1 and 2 and from a live dog of the same kennel with clinical signs of renal failure, as well as frozen renal tissue from dog No. 1 were analysed for melamine content by the Agraquant Melamine Quantitative Kit (Romer Labs ${ }^{\circledR}$, Tulin, Austria). Pet food and renal tissue underwent homogenisation and all samples were purified by Mycosep 224 Aflazon Multifunctional columns (Romer Labs ${ }^{\circledR}$, Tulin, Austria) before analysis. Positive and negative controls were also included. This technique allowed detection of melamine at a minimum concentration of $0.5 \mathrm{ppm}$. The pet food samples were further analysed for aflatoxin A and ochratoxin A1 by AgraQuant ${ }^{\circledR}$ Mycotoxin ELISA kits (Romer Labs $^{\circledR}$, Tulin, Austria), and pesticides by Gas Chromatography with ECD and NPD.

In $\operatorname{dog}$ No. 1 , approximately $70-80 \%$ of the renal tubules showed hydropic degeneration of the epithelial cells, with occasionally foci of coagulative necrosis. Numerous, pale green-brown in colour, round shaped crystals, with radiating striations were evenly distributed throughout the cortex and medulla within renal tubules and collecting ducts (Fig. 2). Some of these crystal structures were arranged in concentric circles. The crystals measured approximately 20 to $80 \mu \mathrm{m}$ in diameter. Larger crystals were more common in the medulla. The crystals demonstrated bright birefringence when viewed under polarized light. The same birefringent crystals were also present in the renal calyces and in urinary bladder. A second type of crystal comprising approximately $10 \%$ of the birefringent crystals was also present within tubule lumens, characterised by different size and shape, and a smoother appearing surface. Some crystals appeared to be in the walls of blood vessels, in addition to the lumens of renal tubules. Low numbers of lymphocytes, plasma cells and multinucleated giant cells multifocally infiltrated renal interstitium. The urinary bladder showed mild diffuse hyperplasia of the transitional epithelium and small, round shaped crystals, with radiating striations were observed within the lumen. The lung showed multifocal alveolar emphysema, and sporadic foci of macrophage alveolar infiltration associated with crystal deposits. Intestine and stomach mucosa was diffusely affected by superficial necrosis with haemorrhages. No evidence of toxic hepatic injury was observed.

Similar renal lesions and crystals were observed on HEstained sections in dog No. 2 however, only approximately $60 \%$ of the renal tubules were affected and mild diffuse interstitial fibrosis was present. Occasional crystals within the renal interstitium elicited a granulomatous inflammatory response with multinucleated giant cells. 

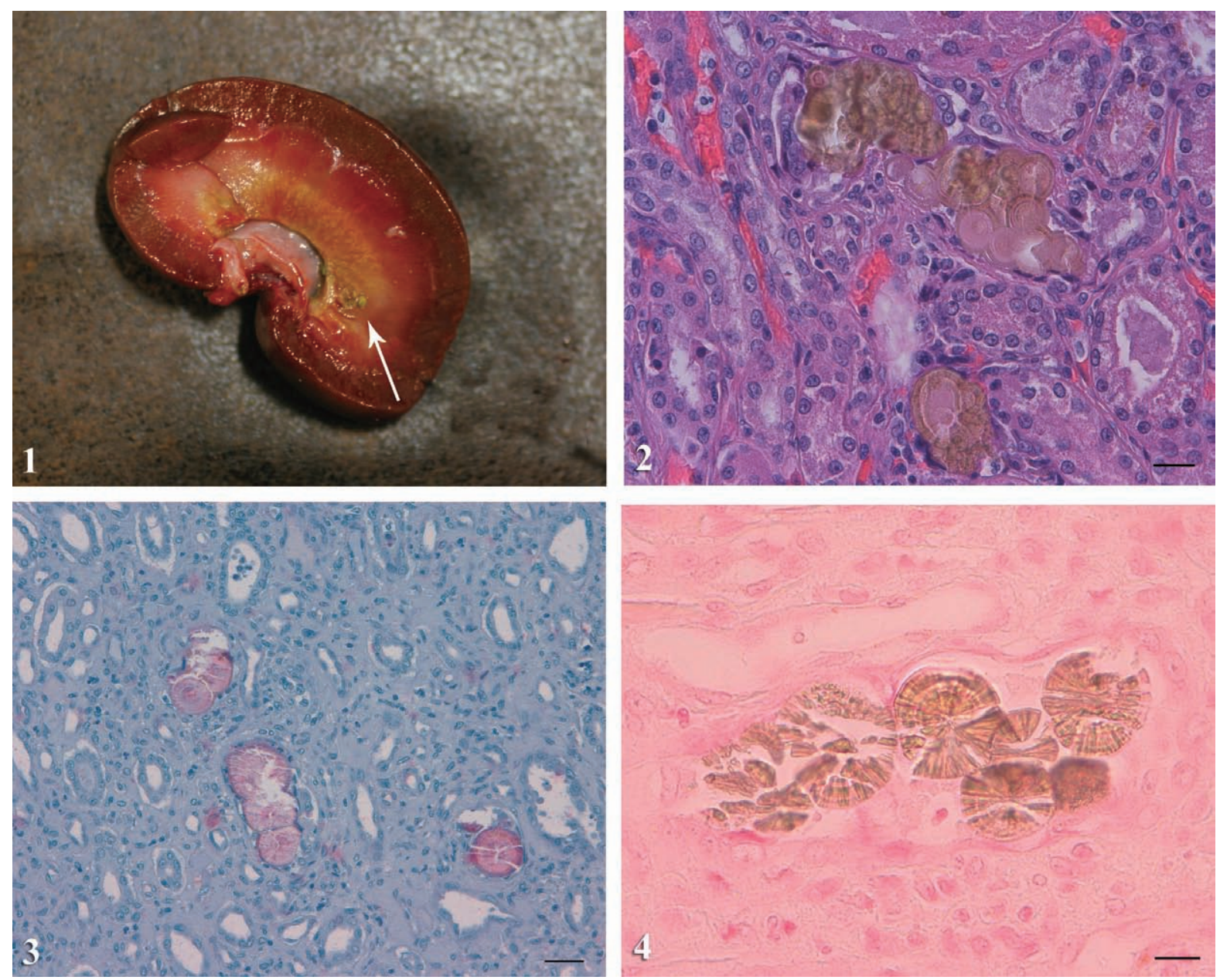

Fig. 1. Kidney of the dog No. 1. Yellowish discolouration of the inner part of the medulla with deposit of green yellowish uroliths (arrow) in the medulla and renal pelvis are evident.

Fig. 2. Renal histological section of dog No. 1 showing large aggregate of pale brown, round mineral crystals within tubules. Tubular epithelium is affected by hydropic degeneration. H\&E. Bar $=20 \mu \mathrm{m}$.

Fig. 3. Kidney of dog No. 1. Pale brown, rough-textured crystals positive stained by Oil Red O stain. Bar=20 $\mu \mathrm{m}$.

Fig. 4. Kidney of dog No. 1. Pale brown, rough crystals not stained by Von Kossa method. Bar=20 $\mu \mathrm{m}$.

In both cases the ORO stain demonstrated variable degrees of positive staining of the pale brown, rough-textured crystals (Fig. 3), indicating a plastic or lipid origin [21], but did not stain the smoother crystals. Von Kossa method stained the smooth crystals, consistent with calcium oxalate, and the mineralised tubular basement membranes, but did not stain the pale brown, rough crystals (Fig. 4). Any bacteriological or virological aetiology, was excluded by laboratory investigations.

$766.8 \mathrm{ppm}$ and $158.5 \mathrm{ppm}$ of melamine were detected in the two analysed pet food, respectively. Melamine was detected in all urine samples at levels of $17 \mathrm{ppm}$ (dog No. 1), $24 \mathrm{ppm}$ (dog No. 2) and $24 \mathrm{ppm}$ (live dog), and in renal tissue at level of $20 \mathrm{ppm}$. No pesticides (LoD: $10 \mathrm{ppb)}$ ) and mycotoxins (LoD: $0.001 \mathrm{mg} / \mathrm{kg}$ aflatoxin A; $0.01 \mathrm{mg} / \mathrm{kg}$ ochratoxin A1) were revealed in pet food.

Potential causes of renal failure in dogs comprise: immu- nological disorders, amyloidosis, neoplasia, nephrotoxicants, renal ischemia, inflammatory or infectious diseases, hereditary and congenital disorders, urinary outflow obstruction, idiopathic. In the cases herein described, the abrupt deposition of crystals within renal tubules and pelvis, associated with diffuse tubular degeneration, was primarily consistent with melamine toxicosis. The predominant crystal type seen in our samples were identical to the crystals observed in the kidneys of animals involved in the 2004 and 2007 melamine associated renal failure epidemic in Asia and US, respectively [2, 20, 23]. In addition, these crystals did not stain with Von Kossa but did stain with Oil Red O. Moreover, as observed in our samples, melamine-containing crystals are predominantly within distal tubules, often exhibit striations, and are easily visualised histologically without polarisation [2].

The combination of the HE appearance, birefringence, 
and histochemical features are quite characteristic of the melamine-containing crystals and distinguish them particularly from calcium oxalate and phosphate $[14,20]$.

Data on the toxicity of melamine in dogs are scarce. In an oral toxicity study in dogs fed $1200 \mathrm{mg}$ melamine/kg b.w. per day for one year, crystalluria was reported after 60 to 90 days and persisted throughout the study period but no other effects were observed [16, 21]. A 4-week oral study was also performed in female dogs applying a lower dose of 126 $\mathrm{mg} / \mathrm{kg}$. In this case no toxicity was reported at the clinical, pathological or histological level $[15,16]$.

The recent incidence of death of cats and dogs in the US has been linked to acute renal failure and investigations are still ongoing to elucidate if the combination of melamine and cyanuric acid may be responsible for the observed toxicity. The specific sensitivity of these two animal species is likely to be explained by melamine and cyanuric acid kinetics parameters rather than being attributable to non-recognized species-specific toxicological effects. It should be noted that cats and dogs physiologically have an acid urine and, dependent of the $\mathrm{pH}$ in urine, melamine will form insoluble complexes with cyanuric acid. This could lead to crystallization and subsequent tissue injury.

Calcium oxalate crystals, observed in both our cases, were a minority of the total number of birefringent crystals. Calcium oxalate dihydrate crystals can be found in the urine of healthy animals and occasionally in cases of ethylene glycol toxicosis. In our cases, there was no history of ethylene glycol exposure. The presence of relatively small numbers of calcium oxalate crystals is most likely the result of secondary oxalosis associated with tubular injury as reported by Brown et al. [2]. Calcium oxalate uroliths are a sequela of metabolic abnormalities that alter the composition of urine and body fluid [13]. Moreover, Maltese and Lhasa Apso are reported to be predisposed to developing urolithiasis [13], and this could have played a role in the onset and severity of the observed pathology.

Toxicologic analyses detected melamine at similar levels in 4 samples ( 1 fresh kidney sample, and 3 urine samples) from 3 different animals. In a previous study [2] toxicologic analyses for detection of melamine and cyanuric acid were performed on 8 samples (4 fresh kidney samples, 2 tissues retrieved from paraffin blocks, and 2 urine samples) from 6 different animals (4 dogs, 2 cats). Melamine and cyanuric acid were detected in all kidney samples and in 1 of 2 urine samples. The animal with negative urine had not been ingesting contaminated pet food for 3 months before its death, so continued excretion of larger detectable amounts of melamine and cyanuric acid would not be expected [2]. In our cases, all the 3 dogs were still feeding contaminated food at the time of urine sampling.

High doses of melamine were identified in two samples of pet-food administered to the dogs for several months (from July to November 2008), with levels of $766.8 \mathrm{ppm}$ and $156.5 \mathrm{ppm}$, respectively. Following the laboratory findings, the Italian Health Authorities carried out further investigation at the manufacturer site and identified melamine in further batches, which were successively recalled. Unfortunately, the origin of melamine contamination remains to be identified. In the 2003 outbreak of acute renal failure in Asia, fungal contamination or mycotoxins (ochratoxin and citrinin) in the pet food were considered [11], since some species of Aspergillus can produce large amounts of oxalates on feedstuffs, causing oxalate nephrosis if ingested [22]. In our case, neither aflatoxin A1 and ochratoxin A1 nor pesticides and infectious agents were detected in the examined pet-food.

Our data, together with those reported from US, seem to indicate that the consumption of melamine-contaminated food could result in nephrotoxicosis in dog. Melamine toxicosis should be considered in case of renal failure onset, particularly in young dogs and cats. Since it has been demonstrated that melamine can be detected in urine, a possible in vivo test for suspected cases should be developed.

To the authors' knowledge, this is the first report of melamine intoxication in dogs in Italy. Furthermore, nephrotoxicosis in Iberian piglets subsequent to exposure to melamine and derivatives in Spain between 2003 and 2006, [10] and the transmission of melamine from feed to cow's milk has been recently demonstrated [8]. These outbreaks emphasize that feedstuffs remain vulnerable to chemical contamination and an increase of surveillance on raw material and animal feed production at EU level is strongly recommended.

\section{REFERENCES}

1. Anonymous. 2007. 104 deaths reported in pet food recall. New York Times, 13; Available from: http://www.nytimes.com/ $2007 / 03 / 28 /$ science/28brfs-pet. html?scp51\&sq5104+deaths+reported+in+pet+food+recall\&st 5nyt. Accessed May 12, 2008.

2. Brown, C.A., Jeong, K., Poppenga, R.H., Puschner, B., Miller, D.M., Ellis, A.E., Kang, K.-Il., Sum, S., Cistola, A.M. and Brown, S.A. 2007. Outbreaks of renal failure associated with melamine and cyanuric acid in dogs and cats in 2004 and 2007. J. Vet. Diagn. Invest. 19: 525-531.

3. Burns, K. 2007a. Witnesses at congressional hearing talk about timing, imports, and surveillance. J. Am. Vet. Med. Assoc. 230: 1601-1602.

4. Burns, K. 2007b. Recall shines spotlight on pet foods. J. Am. Vet. Med. Assoc. 230: 1285-1288.

5. Burns, K., Nolen, S., Kahler, S. and Rezendes, A. 2007. Recall of pet food leaves veterinarians seeking solutions. J. Am. Vet. Med. Assoc. 230: 1128-1129, 1136-1138.

6. Callis, G.M. 2008. Bone. pp. 333-363. In: Theory and Practice of Histological Techniques (Bancroft, J.D. and Gamble, M. eds.), Churchill Livingstone Elsevier, Oxford.

7. Cianciolo, R.E., Bischoff, K., Ebel, J.G., Van Winkle, T.J., Goldstein, R.E. and Serfilippi, L.M. 2008. Clinicopathologic, histologic, and toxicologic findings in 70 cats inadvertently exposed to pet food contaminated with melamine and cyanuric acid. J. Am. Vet. Med. Assoc. 233: 729-737.

8. Cruywagen, C.W., Stander, M.A., Adonis, M. and Calitz, T. 2009. Hot topic: pathway confirmed for the transmission of melamine from feed to cow's milk. J. Dairy Sci. 92: 20462050 . 
9. Dobson, R.L., Motlagh, S., Quijano, M., Cambron, R.T., Baker, T.R., Pullen, A.M., Regg, B.T., Bigalow-Kern, A.S., Vennard, T., Fix, A., Reimschuessel, R., Overmann, G., Shan, Y. and Daston, G.P. 2008. Identification and characterization of toxicity of contaminants of pet food leading to an outbreak of renal toxicity in dogs and cats. Toxicol. Sci. 106: 251-262.

10. González, J., Puschner, B., Pérez, V., Ferreras, M.C., Delgado, L., Muñoz, M., Pérez, C., Reyes, L.E,, Velasco, J., Fernández, V. and García-Marín, J.F. 2009. Nephrotoxicosis in Iberian piglets subsequent to exposure to melamine and derivatives in Spain between 2003 and 2006. J. Vet. Diagn. Invest. 21: 558563.

11. Jeong, W.I., Do, S.H., Jeong, da H., Chung, J.Y., Yang, H.J., Yuan, D.W., Hong, I.H., Park, J.K., Goo, M.J. and Jeong, K.S. 2006. Canine renal failure in three dogs. J. Vet. Sci. 7: 299301.

12. Jones, M.L. 2008. Lipids. pp. 187-215. In: Theory and Practice of Histological Techniques (Bancroft, J.D. and Gamble, M. eds.), Churchill Livingstone Elsevier, Oxford.

13. Langston, C., Gisselman, K., Palma, D. and McCue, J. 2008. Diagnosis of urolithiasis. Comp. Cont. Ed. Vet. 30: 447-454.

14. Lewin-Smith, M.R., Kalasinsky, V.F., Mullick, F.G. and Thompson, M.E. 2009. Melamine-containing crystals in the urinary tracts of domestic animals: sentinel event? Arch. Pathol. Lab. Med. 133: 341-342.

15 Lipschitz, W.L. and Stokey, E. 1945. The mode of action of three new diuretics: melamine, adenine and formoguanamine. J. Pharm. Exp. Ther. 83: 235-249.

16. Organisation for Economic Co-operation and Development. 2002. SIDS Analysis UNEP Publications: Melamine. Avail- able from: http://www.inchem.org/documents/sids/sids/ 108781.pdf. Accessed August 5, 2009.

17. Osborne, C.A., Lulich, J.P., Ulrich, L.K., Koehler, L.A., Albasan, H., Sauer, L. and Schubert, G. 2009. Melamine and cyanuric acid-induced crystalluria, uroliths, and nephrotoxicity in dogs and cats. Vet. Clin. North Am. Small Anim. Pract. 39: $1-14$.

18. Puschner, B., Poppenga, R.H., Loweenstine, L.J., Filigenzi, M.S. and Pesavento, P.A. 2007. Assessment of melamine and cyanuric acid toxicity in cats. J. Vet. Diagn. Invest. 19: 616624.

19. Schmalzried, T.P., Jasty, M., Rosenberg, A. and Harris, W.H. 1993. Histologic identification of polyethylene wear debris using Oil Red O Stain. J. Appl. Biomat. 4: 119-125.

20. Thompson, M.E., Lewin-Smith, M.R., Kalasinsky, V.F., Pizzolato, K.M., Fleetwood, M.L., McElhaney, M.R. and Johnson, T.O. 2008. Characterization of melamine-containing and calcium oxalate crystals in three dogs with suspected pet foodinduced nephrotoxicosis. Vet. Pathol. 45: 417-426.

21. U.S. Food and Drug Administration. 2007. Interim melamine and analogues safety/risk assessment. Available from: http:// www.cfsan.fda.gov/ dms/melamra.html. Accessed August 5, 2009.

22. Wilson, B.J. and Wilson, C.H. 1961. Oxalate formation in moldy feedstuffs as a possible factor in livestock toxic disease. Am. J. Vet. Res. 22: 961-969.

23. Yhee, J.Y., Brown, C.A., Yu, C.H., Kim, J.H., Poppenga, R. and Sur, J.H. 2009. Retrospective study of melamine/cyanuric acid-induced renal failure in dogs in Korea between 2003 and 2004. Vet. Pathol. 46: 348-54. 